\title{
Annotations: A Way to Interoperability in DL
}

\author{
Maristella Agosti and Nicola Ferro \\ Department of Information Engineering - University of Padua \\ \{agosti,ferro\}@dei.unipd.it
}

\begin{abstract}
This paper discusses how annotations and interoperability relate together and affect each other in digital library settings. We analyse interoperability and annotations in the light of the evolution of the field of digital libraries and provide recommendations for successful interoperable annotations towards the European Digital Library.
\end{abstract}

\section{Motivations}

In the beginning, Digital Library (DL) were almost monolithic systems, each one built for a specific kind of information resources - e.g. images or videos and with very specialised functions developed ad-hoc for those contents. This approach caused a flourishing of systems where the very same functions were developed and re-developed many times from scratch. Moreover, these systems were confined to the realm of traditional libraries, since they were the digital counterpart of the latter, and they had a kind of "static" view of their role, which was data-centric rather than user-centric.

Afterwards, DL moved from being monolithic systems to become component and service-based systems, where easily configurable and deployable services can be plugged together and re-used in order to create a DL. Moreover, DL started to be seen as increasingly user-centered systems, where the original content management task is partnered with new communication and cooperation tasks, so that DL become "a common vehicle by which everyone will access, discuss, evaluate, and enhance information of all forms" 7, p. 266]. Finally, DL are no longer perceived as isolated systems but, on the contrary, as systems that need to cooperate together in order to improve the user experience.

In this evolving scenario, the design and development of effective services which foster the cooperation among users and the integration of heterogeneous information resources becomes a key factor. A relevant example of this kind of new services are annotations, i.e. providing users or groups of users with the possibility of adding personal annotations on the managed information resources, even crossing the boundaries of the single DL. Moreover, this push towards valueadd services calls for an unprecedented degree of interoperability among different DL and at various levels, which requires a through understanding and careful design of these complex systems.

The paper discusses how annotations relate to the interoperability issue in DL and how, by exploiting synergies between annotations and interoperability, we can use them as a means to improve the interoperability among different DL.

B. Christensen-Dalsgaard et al. (Eds.): ECDL 2008, LNCS 5173, pp. 291 2952008.

(C) Springer-Verlag Berlin Heidelberg 2008 


\section{$2 \quad$ Interoperability}

The DELOS Network of Excellence on Digital Libraries 11 has been the main driver of evolution in the field of DL in Europe. Two main contributions came out of DELOS with respect to this new vision of DL and the interoperation among them: the DELOS digital library reference model [5, which lays the foundations of DL and facilitates co-operation and interoperability, and the DelosDLMS [1], which is a prototype of the next generation DL system.

The research carried out in DELOS has contributed to developing the vision of the European Digital Library, "a common multilingual access point to Europe distributed digital cultural heritage including all types of cultural heritage institutions" 6]. A relevant project towards this ambitious goal is Europeand2, which is overseen by the EDL Foundation ${ }^{3}$ and deals with the information resources held by European museums, archives, audio-visual archives as well as the issues for making these very different institutions cooperate and interoperate.

In order to support the work toward the "European Digital Library", the European Commission Working Group on Digital Library Interoperability has provided recommendations for both a short term and a long term strategy towards interoperatibility. The working group defines interoperability as "the capability to communicate, execute programs, or transfer data among various functional units in a manner that requires minimal knowledge of the unique characteristics of those units" [6] and identifies six determining factors of it: interoperating entities; objects of interoperation; functional perspective of interoperation; multilinguality; design and user perspective; and interoperability enabling technology.

\section{Annotations}

Throughout our history, annotations have been often used as asynchronous communication tools and as a vehicle for knowledge creation and sharing [2]. Nowadays, in the digital context, annotations come to us as a powerful tool to involve users in approaching DL, to promote the communication and cooperation among user, and to allow us to enhance, enrich, and curate existing content by exploiting user-added information.

Indeed, annotations allow users to naturally merge and link personal contents with the information resources provided by a DL and to create new relationships among existing contents, by means of links that connect annotations together and with existing content. Moreover, annotations and annotated resources constitute a hypertext that can span and cross the boundaries of the single DL and connect information resources that belong and are managed by different DL. In this way, not only annotations foster cooperation among users but they also make DL that otherwise would have been separated cooperate together [4].

${ }^{1}$ http://www.delos.info/

2 http://www . europeana.eu/

3 http://www.europeana.eu/edlnet/edl_foundation/purpose.php 
Therefore, annotations have the potential for contributing to shaping the vision discussed above for the next generation DL.

\section{Impact of Annotations on Interoperability}

The widespread usage of annotations in many different fields and contexts and the familiarity which users have with them make annotations an especially attractive canditate for promoting and improving the interoperability among various DL. Previous research work has already been made part of the DELOS effort towards the next generation and interoperable DL: annotations are part of the DELOS reference model [5] and the Flexible Annotation Service Tool (FAST) has been successfully integrated into DelosDLMS [1].

In the following, we build on our previous results and discuss how annotations can impact on the six determining factors for interoperability discussed above.

- interoperating entities: different cultural heritage institutions - such as libraries, museums, and archives - may desire to offer annotation functionalities on their content for different reasons: for example, libraries and archives may want to promote the enrichment and curation of their content while museums may want to offer their visitors the possibility of recording impressions and observations about the exhibited object and their visit;

- objects of interoperation: annotations need to be modelled and recognized as first class objects in the universe of our interest. Indeed, a clear model of annotation help us to answer the following questions: what does to annotate a digital object mean? What should happen to annotations when instead of digital objects we use their surrogates? How should we deal with annotations when we create aggregate and compound digital objects? How do we insert annotations in the workflow and orchestration of the other functionalities?

These question should be carefully answered since users may expect consistent behaviour with respect to annotations when different DL cooperate together. In addition, users may also expect to exploit annotation as their own way of making different DL interoperate; this would be the case of DL that are not directly cooperating but adopt a common approach to annotations, which can connect objects held by different systems.

- functional perspective of interoperation: this impacts on the way in which an annotation service has to be designed, developed, and made accessible to DL. On the other hand, an annotation service which is able to adopt different protocols, interfaces, and service architectures in order to be integrated into different DL may act as a bridge among those DL if they are not able to cooperate directly. In this way, we can provide a feasible way to interoperability of such different DL, without requiring any modification to them.

- multilinguality: annotations intrinsically entail multilinguality, since they can be written in a language different from the one of the annotated object. This might be a challenge when we design information access systems which deal with multilinguality, since multilingual annotations may be fragmentary or 
short and thus they can require additional flexibility to those systems in order to be effectively processed.

On the other hand, such multilingual annotations can also become an advantage when we deal with multilinguality. Consider the case of a user's query in a language for which there are limited linguistic resources and few specialised information access systems: we might be able to find relevant documents in another language, even without translating the query, if we have annotations in the same language of the initial user's query. This could be a very effective solution when we need to operate in a context where multiple, and possibly not very widely used, languages have to be taken into consideration, as the case of the European Digital Library could be [3].

- design and user perspective: different users may have different expectations from annotations: content providers can consider annotations as a way of making their contents desirable and interesting; content aggregators may wonder how to deal with annotations when they create compound objects; end users may perceive annotations as functional to their own tasks, be they study, research, entertainment, opinion sharing, and so on.

- interoperability enabling technology: this impacts on the way used to design and develop an annotation service. For example, SRU may represent a standardized way of querying and searching by exploiting annotations; OAI-PMH can be a way of sharing and exchanging either whole annotations or metadata about annotations, in the case of complex multimedia annotations; finally, Web services may be an option for exposing the interface of an annotation service.

\section{Acknowledgements}

The authors would like to warmly thank Costantino Thanos, coordinator of DELOS, for his continuous support and advice. The work reported has been partially supported by the TELplus Targeted Project for digital libraries, as part of the eContentplus Program of the European Commission (Contract ECP2006-DILI- 510003).

\section{References}

1. Agosti, M., et al.: DelosDLMS - The Integrated DELOS Digital Library Management System. In: Thanos, C., Borri, F., Candela, L. (eds.) Digital Libraries: Research and Development. LNCS, vol. 4877, pp. 36-45. Springer, Heidelberg (2007)

2. Agosti, M., Bonfiglio-Dosio, G., Ferro, N.: A Historical and Contemporary Study on Annotations to Derive Key Features for Systems Design. IJDL 8(1), 1-19 (2007)

3. Agosti, M., Braschler, M., Ferro, N., Peters, C., Siebinga, S.: Roadmap for MultiLingual Information Access in The European Library. In: Kovács, L., Fuhr, N., Meghini, C. (eds.) ECDL 2007. LNCS, vol. 4675, pp. 136-147. Springer, Heidelberg (2007)

4. Agosti, M., Ferro, N.: A Formal Model of Annotations of Digital Content. ACM TOIS 26(1), 3:1-3:57 (2008) 
5. Candela, L., Castelli, D., Ferro, N., Koutrika, G., Meghini, C., Pagano, P., Ross, S., Soergel, D., Agosti, M., Dobreva, M., Katifori, V., Schuldt, H.: The DELOS Digital Library Reference Model. Foundations for Digital Libraries. ISTI-CNR (2007)

6. Gradmann, S.: Interoperability of Digital Libraries: Report on the work of the EC working group on DL interoperability. In: Seminar on Disclosure and Preservation: Fostering European Culture in The Digital Landscape. National Library of Portugal (2007)

7. Ioannidis, Y., et al.: Digital library information-technology infrastructures. IJDL 5(4), 266-274 (2005) 